\title{
Manifestações atípicas em pacientes com Dengue: revisão de literatura
}

\author{
Atypical manifestations in patients with Dengue: literature review \\ Manifestaciones atípicas en pacientes con Dengue: revisión de la literatura
}

Larissa Toloy Bigaran

ORCID: https://orcid.org/0000-0001-5815-4000 Universidade Brasil, Brasil

E-mail: larissatoloyb@gmail.com

Talita Costa Barbosa

ORCID: https://orcid.org/0000-0002-0396-0651

Universidade Brasil, Brasil

E-mail: talitacostabarbosa@gmail.com

Laiane Cristina Silva da Costa

ORCID: https://orcid.org/0000-0003-2783-7954

Universidade Brasil, Brasil

E-mail: laianecosta2009@hotmail.com

Ana Luisa Bazzo Vieira

ORCID: https://orcid.org/0000-0003-2844-8918 Universidade Brasil, Brasil

E-mail: analuisabazzo@gmail.com

Deleon Ilidio da Silva

ORCID: https://orcid.org/0000-0003-0451-7026

União das Faculdades dos Grandes Lagos, Brasil E-mail: Deleonn_@ @otmail.com

Alana Oda Ribeiro

ORCID: https://orcid.org/0000-0002-4559-7037

União das Faculdades dos Grandes Lagos, Brasil E-mail: alana.oda@hotmail.com

Yasmin Abou Rahal

ORCID: https://orcid.org/0000-0001-6726-3989

União das Faculdades dos Grandes Lagos, Brasil

E-mail: yasmin.rahal@hotmail.com

Beatriz Barboza Garavasso

ORCID: https://orcid.org/0000-0002-6506-4713

União das Faculdades dos Grandes Lagos, Brasil

E-mail: beatriz_garavaso@hotmail.com

Paloma Tonani

ORCID: https://orcid.org/0000-0003-3458-9629

Universidade Brasil, Brasil

E-mail: palomatonani@gmail.com

Kássia Juliana de Almeida Gianini ORCID: https://orcid.org/0000-0003-3984-3753 Universidade Brasil, Brasil

E-mail: kassia_gianini@hotmail.com

Ana Cristina de Souza

ORCID: https://orcid.org/0000-0001-5329-838X Universidade Brasil, Brasil

E-mail: tininha_2106@hotmail.com

Herlen Márcio Dourado Alves Farias ORCID: https://orcid.org/0000-0001-7078-2472 Universidade Brasil, Brasil

E-mail: hmdafarias@gmail.com

Olivia Mendes Amendola

ORCID: https://orcid.org/0000-0002-1672-1019 Universidade Brasil, Brasil

E-mail: olivia_amendola159@hotmail.com

Elenberg Chaves de Paula

ORCID: https://orcid.org/0000-0003-4170-7726

União das Faculdades dos Grandes Lagos, Brasil

E-mail: elenbergde@hotmail.vcom

Elizabete Santos Melo

ORCID: https://orcid.org/0000-0002-7322-9370 Universidade Brasil, Brasi

E-mail: enfaelizabetemelo@gmail.com 


\begin{abstract}
Resumo
A dengue é um grande problema de saúde pública, o qual afeta principalmente a população das regiões tropicais e subtropicais no mundo, além dos milhares de viajantes que visitam essas áreas. A transmissão ocorre através da picada dos vetores fêmeas do Aedes aegypti, Aedes albopictus, Aedes scutellari ou Aedes polynesiensis, infectados pelo vírus, podendo levar a manifestações desde assintomática a febre hemorrágica. Dessa maneira, o estudo realizado é uma revisão integrativa da literatura, descrita como um método de pesquisa que permite a busca, a avaliação crítica e a síntese das evidências disponíveis do tema investigado, sendo utilizado vinte artigos para realização dele. Assim, a dengue pode ter sua manifestação por diversas maneiras, desde infecção assintomática a febre indiferenciada, dengue hemorrágica ou dengue por síndrome de choque. Logo, ressalta-se a importância do conhecimento detalhado de todas as manifestações que pode acometer o paciente com dengue, além de executar um exame clínico bem detalhado, fazendo uso de meios complementares para diagnóstico, tais como exames específicos. O tempo é fundamental nessa hora, visto que pode favorecer o prognóstico do paciente, evitando futuras complicações.
\end{abstract}

Palavras-chave: Dengue; Manifestações incomuns; Aedes; Vírus da Dengue.

\begin{abstract}
Dengue is a major public health problem, which mainly affects the population of tropical and subtropical regions in the world, in addition to the thousands of travelers who visit these areas. Transmission occurs through the bite of female vectors of Aedes aegypti, Aedes albopictus, Aedes scutellari or Aedes polynesiensis, infected by the virus, which can lead to manifestations ranging from asymptomatic to hemorrhagic fever. Thus, the study carried out is an integrative literature review, described as a research method that allows for the search, critical evaluation and synthesis of available evidence on the topic investigated, being used twenty articles to carry out the same. Thus, dengue can be manifested in different ways, from asymptomatic infection to undifferentiated fever, dengue hemorrhagic fever or dengue shock syndrome. Therefore, the importance of detailed knowledge of all manifestations that can affect the patient with dengue is highlighted, in addition to performing a very detailed clinical examination, making use of complementary means for diagnosis, such as exams. Time is essential at this time, as it can favor the patient's prognosis, avoiding future complications.
\end{abstract}

Keywords: Dengue; Unusual manifestations; Aedes; Dengue virus.

\title{
Resumen
}

El dengue es un importante problema de salud pública, que afecta principalmente a la población de las regiones tropicales y subtropicales del mundo, además de a los miles de viajeros que visitan estas áreas. La transmisión se produce por la picadura de hembras vectores de Aedes aegypti, Aedes albopictus, Aedes scutellari o Aedes polynesiensis, infectadas por el virus, que pueden dar lugar a manifestaciones que van desde fiebre asintomática a hemorrágica. Así, el estudio realizado es una revisión integradora de la literatura, descrita como un método de investigación que permite la búsqueda, evaluación crítica y síntesis de la evidencia disponible sobre el tema investigado, utilizándose veinte artículos para realizar la misma. Así, el dengue puede manifestarse de diferentes formas, desde una infección asintomática hasta fiebre indiferenciada, dengue hemorrágico o síndrome de choque por dengue. Por ello, se destaca la importancia del conocimiento detallado de todas las manifestaciones que pueden afectar al paciente con dengue, además de realizar un examen clínico muy detallado, haciendo uso de medios complementarios para el diagnóstico, como los exámenes. El tiempo es fundamental en este momento, ya que puede favorecer el pronóstico del paciente, evitando futuras complicaciones.

Palabras clave: Dengue; Manifestaciones inusuales; Aedes; Virus del Dengue.

\section{Introdução}

A infecção viral por dengue coloca em perigo cerca de 2,5 bilhões de pessoas que vivem em regiões tropicais e subtropicais do mundo e milhares de viajantes sorologicamente ingênuos visitam essas áreas anualmente, colocando uma parte significativa da população humana em risco de infecção (Estofolete et al., 2019). Esta acontece pela picada dos vetores fêmeas do Aedes aegypti, Aedes albopictus, Aedes scutellari ou Aedes polynesiensis, infectados pelo vírus - que é envolto por lipídio, com um genoma composto por uma molécula de RNA de fita simples de sentido positivo - do gênero Flavavírus, da família dos Flaviviridae, pode levar a manifestações clínicas que variam desde assintomática a febre hemorrágica (Mallhi et al., 2015; Mota et al., 2017).

Existem quatro sorotipos: DEN-1, DEN-2, DEN-3, DEN-4 e a infecção por um deles não confere proteção cruzada. Normalmente, a doença cursa com sintomas que incluem febre alta e de início abrupto, frequentemente, acompanhada de cefaléia intensa, dor retro-orbitária, dores musculares, articulares e abdominal, diarreia, náuseas, vômitos, inapetência e exantema máculo-papular acompanhado ou não de prurido, de forma que, em sua maioria, a doença é autolimitada e com baixa 
mortalidade. Todavia, em desfechos mais graves observar-se a febre hemorrágica da dengue (graus 3 e 4) e a Síndrome do Choque da Dengue - esta caracterizada por vazamento vascular e falência de múltiplos órgãos - ambas podendo levar à morte (Umakanth \& Sughantan, 2020; Vita et al., 2009). Ademais, mostraram-se relevantes, como fatores preditivos de mau prognóstico, os achados laboratoriais de leucocitose, de plaquetopenia e o aumento das transaminases, além dos sinais e sintomas: dor abdominal intensa e contínua, vômitos incoersíveis, hipotensão postural e arterial, manifestações hemorrágicas e extravasamento plasmático (Souza et al., 2009).

A "Síndrome da Dengue Expandida" é uma nova entidade adicionada em 2011 ao sistema de classificação da Organização Mundial da Saúde, que incorporou o amplo espectro de manifestações incomuns dessa patologia, com o acometimento de diversos órgãos, incluindo fígado, rins, coração e cérebro (Mallhi et al., 2015). Um estudo prospectivo com 175 pacientes realizado entre 2011 e 2013, demonstrou que manifestações incomuns e raras da dengue foram documentadas em 115 pacientes (66\%); dentre elas estão: hepatite (70\% dos casos); derrame pleural (11\% dos casos); insuficiência renal aguda (10\% dos casos); complicações neurológicas como encefalite ( $7,4 \%$ dos casos); miocardite ( $9 \%$ dos casos) e sangramento de úlceras gástricas (3,4\% dos casos). O endotélio é o alvo dos mecanismos imunopatológicos na dengue e a permeabilidade vascular e os distúrbios de coagulação são marcas registradas da doença; esses mecanismos podem explicar o envolvimento sistêmico variado que ocorre em diversos casos (Neeraja et al., 2014).

Desse modo, é importante que os médicos e profissionais da saúde estejam cientes desses problemas que podem estar subnotificados e, a falta de conhecimento entre os médicos primários, pode comprometer o diagnóstico clínico, o que impacta no desfecho da doença nos contaminados - já que o período de janela terapêutica dos casos graves é muito curto. Assim, se torna essencial a atenção e o monitoramento das manifestações incomuns e raras, para melhorar o manejo e a evolução clínica dos pacientes (Kularatne et al., 2018).

\section{Metodologia}

O estudo realizado é uma revisão integrativa da literatura, descrita como um método de pesquisa que permite a busca, a avaliação crítica e a síntese das evidências disponíveis do tema investigado (Mendes et al., 2008). Os descritores utilizados foram "unusual manifestations" and "dengue". Foram encontrados dois mil e quarenta e seis (2046) artigos e, desses, selecionados vinte (20), nos idiomas inglês e português. Para alcance dos estudos escolhidos, as plataformas usadas foram PubMed, SciELO, MEDLINE e Google Scholar.

Em relação aos critérios de inclusão e exclusão, utilizou-se como critérios de inclusão: artigos disponíveis com publicação nos idiomas português e inglês que abordassem o tema do estudo, de acordo com o título; já como exclusão, não foram selecionados os textos que citavam outras arboviroses.

\section{Resultados e Discussão}

Através das buscas efetivadas nas bases de dados, encontraram-se 103 artigos na PubMed, 02 artigos na SciELO, 62 artigos na MEDLINE e 1860 no Google Scholar, totalizando 2046 artigos que, após aplicação dos critérios de inclusão e exclusão, reduziram-se a 20 documentos, os quais foram utilizados para a confecção da presente revisão; sendo eles, 10 artigos retirados da base PubMed, 01 da base SciELO, 06 da base MEDLINE e 03 da base Google Scholar, nos idiomas inglês e português.

Para melhor esclarecimento dos artigos encontrados e utilizados, optou-se pelo uso do Quadro 1 com os dados: autores e ano, título, objetivo e delineamento, utilizados a fim de organizar e demonstrar os dados colhidos. 
Quadro 1 - Artigos utilizados para a revisão.

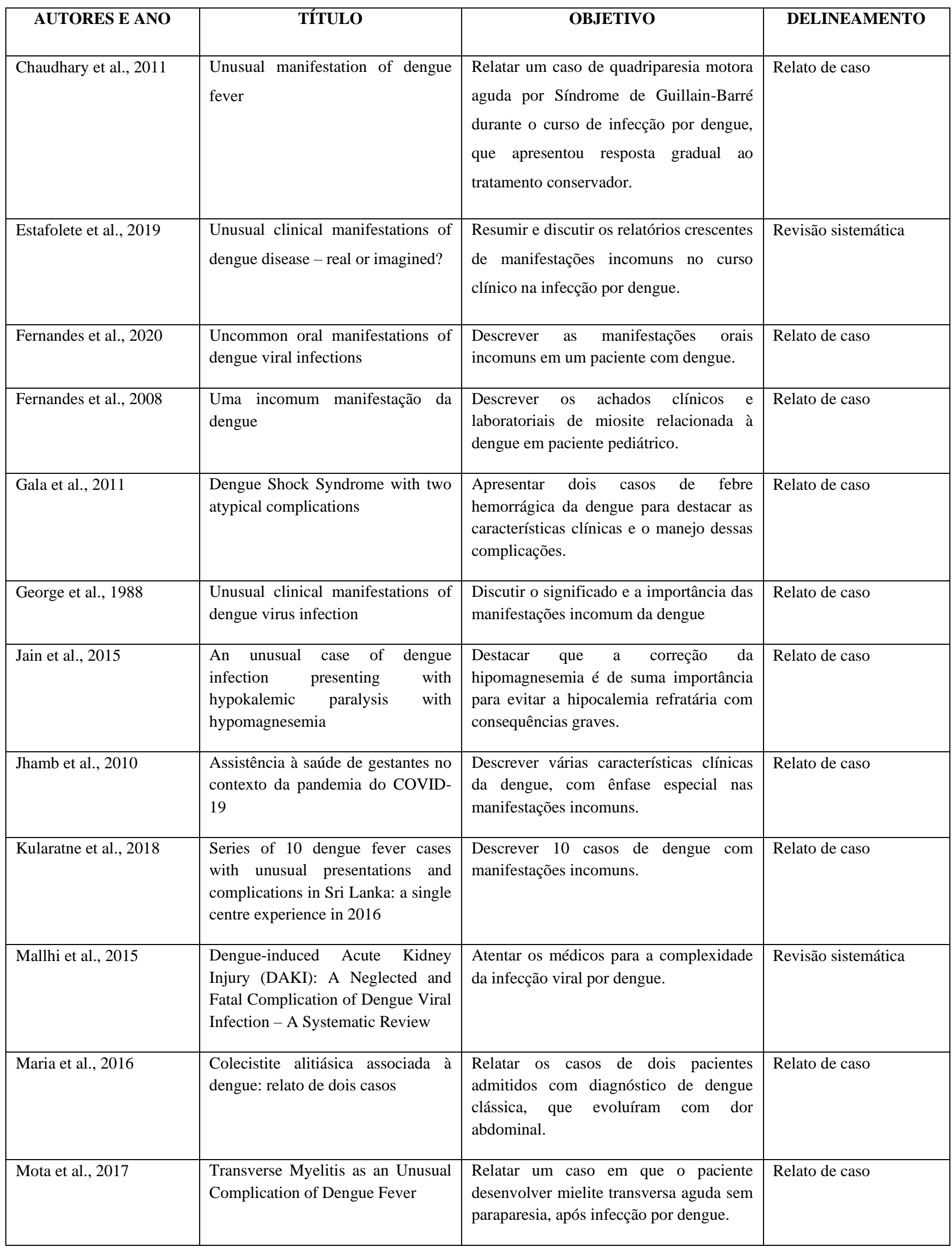




\begin{tabular}{|c|c|c|c|}
\hline Neeraja et al., 2014 & $\begin{array}{l}\text { Unusual and rare manifestations of } \\
\text { dengue during a dengue outbreak in } \\
\text { a tertiary care hospital in South } \\
\text { India }\end{array}$ & $\begin{array}{l}\text { Documentar as manifestações raras da } \\
\text { dengue em } 175 \text { pacientes hospitalizados. }\end{array}$ & Estudo prospectivo \\
\hline Patey et al., 1993 & $\begin{array}{l}\text { Unusual Neurologic Manifestations } \\
\text { Occurring during Dengue Fever } \\
\text { Infection }\end{array}$ & $\begin{array}{l}\text { Relatar dois casos de pacientes jovens } \\
\text { que tiveram manifestações neurológicas } \\
\text { incomuns após infecção por dengue. }\end{array}$ & Relato de caso \\
\hline Porwal \& Chauhan., 2016 & Dengue Encephalitis & Relatar um caso de encefalite por dengue. & Relato de caso \\
\hline Serufo et al., 2000 & Dengue: uma nova abordagem & $\begin{array}{l}\text { Revisar os conceitos de dengue clássico, } \\
\text { com ou sem hemorragia, e de febre } \\
\text { hemorrágica do dengue, que pode cursar } \\
\text { sem fenômenos hemorrágicos, com ou } \\
\text { sem síndrome do choque de dengue. }\end{array}$ & Artigo de opinião \\
\hline $\begin{array}{l}\text { Sirivichayakul et al., } \\
2000\end{array}$ & $\begin{array}{l}\text { Dengue infections with unusual } \\
\text { manifestations: a case report }\end{array}$ & $\begin{array}{l}\text { Relatar um caso de paciente com dengue } \\
\text { hemorrágica grau } 2 \text { com insuficiência } \\
\text { hepática e encefalopatia hepática e } \\
\text { elevação das enzimas hepáticas. }\end{array}$ & Relato de caso \\
\hline Souza et al., 2009 & $\begin{array}{l}\text { Colecistite alitiásica por dengue: } \\
\text { relatos de casos }\end{array}$ & $\begin{array}{l}\text { Destacar a importância de investigar a } \\
\text { colecistite alitiásica em pacientes com } \\
\text { dengue, que tenham dor abdominal como } \\
\text { sinal de alerta, através da exposição de } \\
\text { dois casos diagnosticados no Cento de } \\
\text { Referência da Dengue (CRD) no } \\
\text { município de Campos dos Goytacazes no } \\
\text { primeiro semestre de } 2007 \text {. }\end{array}$ & Relato de caso \\
\hline $\begin{array}{l}\text { Thisyakorn \& } \\
\text { Thisyakorn, } 1994\end{array}$ & $\begin{array}{l}\text { Dengue infection with unusual } \\
\text { manifestations }\end{array}$ & $\begin{array}{l}\text { Relatar manifestações atípicas em } \\
\text { pacientes internados no departamento de } \\
\text { pediatria em Bangcoc. }\end{array}$ & Relato de caso \\
\hline $\begin{array}{l}\text { Umakanth \& Suganthan, } \\
2020\end{array}$ & $\begin{array}{l}\text { Unusual Manifestations of Dengue } \\
\text { Fever: A review on Expanded } \\
\text { Dengue Syndrome }\end{array}$ & $\begin{array}{l}\text { Fornecer conhecimento sobre a síndrome } \\
\text { de dengue expandida, que ajuda a detectar } \\
\text { o diagnóstico de dengue precocemente. }\end{array}$ & Revisão sistemática \\
\hline
\end{tabular}

Fonte: Elaborado por autores.

A dengue é uma doença arboviral, mais prevalente em regiões tropicais e subtropicais, causado por quatro sorotipos. Sua apresentação pode ter diversas maneiras, de infecção assintomática até febre indiferenciada, dengue, dengue hemorrágica ou dengue por síndrome de choque. As suas manifestações podem ser neurológicas, embora sejam mais raras, podendo incluir encefalopatia, meningoencefalite, miosite, rabdomiólise, mielite, acidente vascular cerebral, paralisia hipocalêmica, Síndrome Guillain Barré, papiledemia, encefalomielite disseminada aguda, neuromielite óptica e neurite óptica. (Jain et al., 2015).

Em caso suspeito de dengue, com doença febril aguda, duração máxima de sete dias, tem a manifestação de pelo menos dois dos seguintes sinais ou sintomas: cefaleia, dor retro orbitária, mialgia, artralgia, prostração ou exantema, associados ou não a hemorragias, dor abdominal persistente e intensa, vômitos, hipotensão postural, sangramento de mucosas, hepatomegalia dolorosa, queda abrupta de plaquetas e aumento repentino de hematócrito representam sinais de alarme e indicam maior gravidade do caso (Maria et al., 2016).

Segundo as diretrizes da Organização Mundial da Saúde, também classificam a dengue como Síndrome da Dengue expandida para incorporar um amplo espectro de manifestações incomuns de infecção por dengue que afetam vários sistemas, tais como fígado, coração, rim, cérebro. (Mallhi et al., 2015). 
Ultimamente tem havido um crescente número de casos notificados de infecção por dengue com manifestações incomuns e na sua grande maioria deles tinha a síndrome do choque da dengue, o qual pode ter o acometimento hepático com insuficiência hepática e encefalopatia hepática e elevação muito elevada das enzimas hepáticas. A sua recuperação completa é alcançada após terapia conservadora (Sirivichayakul et al., 2018).

De acordo com alguns estudos, uma complicação decorrente da dengue pode ser miosite aguda benigna, caracterizada pela intensa mialgia com comprometimento de panturrilhas, as vezes coxas. A dor é percebido após período de repouso, principalmente ao acordar. Pode ter impacto na marcha, com claudicação e até impossibilidade de deambular. O seu aparecimento é depois dos sintomas virais estarem desaparecendo. O prognóstico é favorável, com recuperação total após um a cinco dias ou até quatro semanas. Os exames revelam um hemograma normal, as vezes com leucopenia, hemoglobinúria, aumento da CPK, alterações eletromiográficas. A explicação para tal acometimento são a invasão direta por partículas virais ou o dano muscular por mediação imunológica desencadeada por vírus. Insuficiência renal aguda, distúrbios eletrolíticos e síndrome compartimental podem se desenvolver na presença de rabdomiólise (Fernandes et al., 2008).

Outra complicação que pode ser observada é hepatite fulminante, encefalopatia, hepatite com insuficiência renal. Diante disso, vale ressaltar a importância de uma avaliação clínica completa, com análise de eletrólitos, marcadores, enzimas, além do exame clínico (George et al., 1988).

Além disso, pode ter acometimento na vesícula biliar. A colecistite alitiásica é uma manifestação atípica da dengue, normalmente é autolimitada, o qual deve ser pesquisada em todos os pacientes que tenham dor abdominal, como um sinal de alerta e a conduta adequada restringe-se ao tratamento de suporte, devendo a cirurgia ser reservada às complicações (Souza et al., 2009).

\section{Conclusão}

A dengue é um problema de saúde pública, acometendo grande parte dos países nas regiões tropicais e subtropicais. Com o advento das viagens, da urbanização descontrolada e dos movimentos urbanos massivos, houve a ampla circulação de vários sorotipos em todo o mundo. As diretrizes criadas foram para qualificar casos graves de dengue no início do curso da infecção, com o propósito de evitar complicações clínicas graves - como encefalite, miocardite, hepatite, insuficiência renal, entre outros. Assim, o diagnóstico preciso de dengue em pacientes com expressão incomum da doença é bastante desafiador. À medida que essas manifestações não são diagnosticadas, podem aumentar a taxa de morbimortalidade por dengue, principalmente quando os órgãos principais são acometidos. Logo, uma descrição detalhada das manifestações dessa patologia é fundamental para aprimorar o conhecimento dos profissionais e auxiliar a conduta terapêutica necessária. Os médicos devem estar atentos a essas características incomuns para que possam diagnosticar a dengue precocemente, especialmente durante epidemias em andamento.

Para trabalhos futuros sugere-se o incentivo à publicação de relatos de casos sobre manifestações atípicas da dengue, de modo que haja a difusão das mesmas entre a comunidade médica e, com isso, facilite-se o diagnóstico precoce da doença, favorecendo o desfecho do quadro clínico dos pacientes.

\section{Referências}

Chaudhary, S. C., Mohanty, D., Sonkar, S. K., Gupta, D. K., \& Gupta, A. (2011). Unusual manifestation of dengue fever. Case Reports, 2011 (jun23 1), bcr0220113839-bcr0220113839. https://doi.org/10.1136/bcr.02.2011.3839

Célia, R., Caroline, R. T., \& Priscila, B. B. (2021). Uma incomum manifestação da dengue. Revista Científica Da Faculdade de Medicina de Campos, 3(1), 13-16. http://www.fmc.br/ojs/index.php/RCFMC/article/view/147 
Estofolete, C. F., de Oliveira Mota, M. T., Bernardes Terzian, A. C., de Aguiar Milhim, B. H. G., Ribeiro, M. R., Nunes, D. V., Mourão, M. P., Rossi, S. L., Nogueira, M. L., \& Vasilakis, N. (2019). Unusual clinical manifestations of dengue disease - Real or imagined? Acta Tropica, 199, 105134. https://doi.org/10.1016/j.actatropica.2019.105134

Europe PMC. (2016). Europe PMC. Europepmc.org. https://europepmc.org/article/med/26577971

Europe PMC. (2016). Europe PMC. Europepmc.org. https://europepmc.org/article/med/26209406

Fernandes, C. I. R., Perez, L. E. da C., \& Perez, D. E. da C. (2020). Uncommon oral manifestations of dengue viral infection. Brazilian Journal of Otorhinolaryngology, 86, 3-5. https://doi.org/10.1016/j.bjorl.2016.10.001

Fernandes., et al. (2008) Uma incomum manifestação da dengue . Revista Científica da FMC, 3 (1),13-16 .

Gala, H. C., Avasthi, B. S., \& Lokeshwar, M. R. (2011). Dengue Shock Syndrome with Two Atypical Complications. The Indian Journal of Pediatrics, 79(3), 386-388. https://doi.org/10.1007/s12098-011-0551-5

Jhamb, R., Kumar, A., Ranga, G. S., \& Rathi, N. (2011). Unusual manifestations in dengue outbreak 2009, Delhi, India. The Journal of Communicable Diseases, 42(4). https://pubmed.ncbi.nlm.nih.gov/22471194/

Kularatne, S. A. M., Ralapanawa, U., Dalugama, C., Jayasinghe, J., Rupasinghe, S., \& Kumarihamy, P. (2018). Series of 10 dengue fever cases with unusual presentations and complications in Sri Lanka: a single centre experience in 2016. BMC Infectious Diseases, 18(1). https://doi.org/10.1186/s12879-018-3596-5

Mallhi, T. H., Sarriff, A., Adnan, A. S., Khan, Y. H., Hamzah, A. A., Jummaat, F., \& Khan, A. H. (2015). Dengue-induced Acute Kidney Injury (DAKI): A Neglected and Fatal Complication of Dengue Viral Infection--A Systematic Review. Journal of the College of Physicians and Surgeons--Pakistan : JCPSP, 25(11). https://pubmed.ncbi.nlm.nih.gov/26577971/

Maria, G., Thaysa, D. P., Marques, N. O., Lima, R. S., \& Lima, S. (2016). Colecistite alitiásica associada à dengue: relato de dois casos. 26(0), S149-S151. http://www.rmmg.org/artigo/detalhes/2015

Mota, M. T. de O., Estofolete, C. F., Zini, N., Terzian, A. C. B., Gongora, D. V. N., Maia, I. L., \& Nogueira, M. L. (2016). Transverse Myelitis as an Unusual Complication of Dengue Fever. The American Journal of Tropical Medicine and Hygiene, 96(2), 380-381. https://doi.org/10.4269/ajtmh.16-0284

Neeraja, M., lakshmi, V., Teja, V. D., Lavanya, V., Priyanka, E. N., Subhada, K., Parida, M. M., Dash, P. K., Sharma, S., Rao, P. V. L., \& Reddy, G. (2014). Unusual and rare manifestations of dengue during a dengue outbreak in a tertiary care hospital in South India. Archives of Virology, 159(7), 1567-1573. https://doi.org/10.1007/s00705-014-2010-x

Patey, O., Lafaix, C., Breuil, J., \& Ollivaud, L. (1993). Unusual Neurologic Manifestations Occurring during Dengue Fever Infection. The American Journal of Tropical Medicine and Hygiene, 48(6), 793-802. https://doi.org/10.4269/ajtmh.1993.48.793

Porwal, J., \& Chauhan, A. (2016). Dengue Encephalitis. The Journal of the Association of Physicians of India,64(8). https://pubmed.ncbi.nlm.nih.gov/27762126/

Serufo, J. C., Nobre, V., Rayes, A., Marcial, T. M., \& Lambertucci, J. R. (2000). Dengue: uma nova abordagem. Revista Da Sociedade Brasileira de Medicina Tropical, 33(5), 465-476. https://doi.org/10.1590/s0037-86822000000500008

Sirivichayakul, C., Sabcharoen, A., Chanthavanich, P., Pengsaa, K., Chokejindachai, W., \& Prarinyanupharb, V. (2018). Dengue infection with unusual manifestations: a case report. Journal of the Medical Association of Thailand = Chotmaihet Thangphaet, 83(3). https://pubmed.ncbi.nlm.nih.gov/10808688/

Souza, L. J. de., Zagne, S. M. O., Araújo, P. G., Zagne, L. O., Maciel, N. de S., \& Araujo, N. F. (2009). EDITORIAL 1 A Sociedade Brasileira de Clínica Médica e os 20 anos de existência Brazilian Society of Internal Medicine and 20 years existence 11 Dengue: alertas clínicos e laboratoriais da evolução grave da doença Dengue: clinical and laboratory alerts of the evolution of the serious illness. https://www.sbcm.org.br/revistas/RBCM/RBCM-200901.pdf\#page $=55$

Thisyakorn, U., \& Thisyakorn, C. (2020). Dengue infection with unusual manifestations. Journal of the Medical Association of Thailand = Chotmaihet Thangphaet, 77(8). https://pubmed.ncbi.nlm.nih.gov/7876762/

Umakanth, M., \& Suganthan, N. (2020). Unusual Manifestations of Dengue Fever: A Review on Expanded Dengue Syndrome. Cureus. https://doi.org/10.7759/cureus. 10678 